\title{
Formas de tratamiento pronominales en el español de Cali, Colombia*
}

\author{
RÓBINSON GRAJALES ALZATE** \\ MARGARETH MARMOLEJO CAICEDO ${ }^{* * *}$
}

Recepción: 12 de abril de 2020

Aprobación: 14 de junio de 2021

Forma de citar este artículo: Grajales, R \& Marmolejo, M. (2021). Formas de tratamiento pronominales en el español de Cali, Colombia, Cuadernos de Lingüística Hispánica, (37), e12694

(di) https://doi.org/10.19053/0121053X.n37.2021.12694

* Este artículo es derivado de la investigación Estudio sociolingüístico del habla de Cali, financiado por la Vicerrectoría de Investigaciones de la Universidad del Valle, código C.I 4379, y ejecutado entre el 11 de febrero de 2019 y el 18 de diciembre de 2020 .

** Doctor en Lingüística de la Universidad de Antioquia. Profesor de Lingüística Teórica y Descriptiva de la Escuela de Ciencias del Lenguaje de la Universidad del Valle, Colombia. Actualmente es el coordinador del equipo PRESEEA-Cali y dirige el proyecto Estudio Sociolingüístico de Cali. Además, es director del Grupo de Estudios Lingüísticos e Interdisciplinarios de la Universidad del Valle, en el que se trabajan las líneas de investigación en lingüística hispánica, sociolingüística y lexicografía. robinson.grajales@correunivalle.edu.co@https://orcid. org/0000-0002-8699-1321

*** Magíster en Estudios Interlingüísticos e Interculturales de la Universidad del Valle. Docente de la Universidad del Valle. Actualmente es investigadora del equipo PRESEEA-Cali y pertenece al Grupo de Estudios Lingüísticos e Interdisciplinarios de la Universidad del Valle y al Grupo de Estudios Sociolingǘísticos de la Universidad de Antioquia. margareth.marmolejo@correounivalle.edu.co @ https://orcid.org/0000-0003-4146-6125 


\section{Resumen}

Esta investigación sobre el uso de las formas de tratamiento pronominales en Cali, Colombia, busca describir la distribución de su uso en relación con las variables sexo, edad, nivel educativo y estrato socioeconómico; establecer si estas variables son significativas en la elección de la forma pronominal según el vínculo con el interlocutor; determinar si los resultados indican una tendencia de tratamiento de distancia o de solidaridad; y contrastar los resultados con otros estudios colombianos sobre este fenómeno. Los datos se recogieron a través de la adaptación del cuestionario sociolingüístico propuesto por PRESEEA. En total se obtuvieron las respuestas de 72 informantes sobre la elección de las formas de tratamiento pronominales según la relación con el interlocutor. A estos datos se les aplicó un análisis estadístico descriptivo e inferencial que arrojó, entre otros, que en Cali prevalece el uso de usted frente a tú y vos, lo cual indica una tendencia a la cortesía de distanciamiento; no obstante, la cortesía de solidaridad es impulsada por las generaciones jóvenes, los niveles educativos altos y los estratos socioeconómicos medios; la variable sexo es significativa en la elección de forma de tratamiento según el sexo del interlocutor y en las relaciones no familiares.

Palabras clave: cortesía verbal, sociolingüística, formas de tratamiento pronominales, PRESEEA, español de Colombia.

\section{Pronominal Treatment Forms in the Spanish spoken in Cali, Colombia}

\section{Abstract}

This research on the use of pronominal treatment forms in Cali, Colombia, seeks to describe the distribution of their use in relation to sex, age, educational level, and socioeconomic stratum variables; establish if these variables are significant in the choice of the pronominal form according to the link with the interlocutor; determine if the results indicate a trend of distance or solidarity treatment; and contrast the results with other Colombian studies on this topic. Data were collected through adaptation of the sociolinguistic questionnaire proposed by PRESEEA. In total, responses of 72 informants were obtained on the choice of pronominal treatment forms according to the relationship with the interlocutor. A descriptive and inferential statistical analysis was applied to these data, that showed, among others, that in Cali the use of the formal you (usted), prevails over the informal ones (vos and tú), which indicates a tendency towards distancing courtesy; however, the courtesy of solidarity is promoted by the younger generations, the high educational levels and the middle socioeconomic strata; sex as variable is significant in the choice of the treatment form according to the sex of the interlocutor and in non-family relationships.

Keywords: verbal courtesy, sociolinguistics, pronominal treatment forms, PRESEEA, Colombian Spanish. 


\section{Formes de traitement pronominaux en espagnol de Cali, Colombie}

\section{Résumé}

Cette recherche sur l'utilisation des formes de traitement pronominales à Cali, Colombie, cherche à décrire la distribution de leur emploi en rapport avec les variables de sexe, âge, niveau de formation et niveau socio-économique; à établir si ces variables sont significatives dans le choix de la forme pronominale selon le lien avec l'interlocuteur; à déterminer si les résultats indiquent une tendance de traitement de distanciation ou de solidarité; et à comparer les résultats avec d'autres études colombiennes sur ce phénomène. Les données ont été recueillies grâce à l'adaptation du questionnaire sociolinguistique proposé par PRESEEA. Au total, on a obtenu les réponses de 72 participants à l'enquête concernant le choix des formes de traitement pronominales selon la relation avec l'interlocuteur. On a soumis ces données à une analyse statistique descriptive et inférentielle qui a montré, entre autres, qu'à Cali l'usage de vous prévaut sur l'utilisation de toi et de "vos", ce qui indique une tendance à la courtoisie de distanciation. Néanmoins, la courtoisie de solidarité est encouragée par les générations de jeunes, les niveaux de formation élevés et les couches socioéconomiques moyennes; la variable sexe est significative dans le choix de la forme de traitement selon le sexe de l'interlocuteur et dans les relations non familiales.

Mots-clés: courtoisie verbale, sociolinguistique, formes de traitement pronominales, PRESEEA, espagnol de Colombie.

\section{Formas pronominais de tratamento no espanhol de cali, colômbia}

\section{Resumo}

Esta pesquisa sobre 0 uso de formas de tratamento pronominais em Cali, Colômbia, busca descrever a distribuição de seu uso em relação às variáveis sexo, idade, escolaridade e estrato socioeconômico; estabelecer se essas variáveis são significativas na escolha da forma pronominal de acordo com 0 vínculo com 0 interlocutor; determinar se os resultados indicam tendência de distanciamento ou tratamento solidário; e contrastar os resultados com outros estudos colombianos sobre este fenômeno. Os dados foram coletados por meio da adaptação do questionário sociolinguístico proposto pelo PRESEEA. No total, foram obtidas as respostas de 72 informantes sobre a escolha das formas de tratamento pronominais de acordo com o relacionamento com 0 interlocutor. A estes dados foi aplicada uma análise estatística descritiva e inferencial que mostrou, entre outros, que em Cali o uso de "usted" prevalece sobre "tu" e "vos", o que indica uma tendência ao distanciamento da cortesia; No entanto, a cortesia da solidariedade é promovida pelas gerações mais jovens, pelos níveis educacionais elevados e pelos estratos socioeconômicos médios; a variável sexo é significativa na escolha da forma de tratamento de acordo com o sexo do interlocutor e nas relações não familiares.

Palavras-chave: cortesia verbal, sociolinguística, formas pronominais de tratamento, PRESEEA, espanhol colombiano. 


\section{Introducción}

Las formas de tratamiento son un conjunto de signos referenciales de los que dispone un hablante para dirigirse a su interlocutor (Bertolotti, 2015). Dentro de este conjunto se encuentran las formas de tratamiento pronominales (FTP) que corresponden al sistema pronominal de segunda persona en español: tú, usted, vos (Fontanella, 1999) y sumercé (Cepeda, 2014). Estas FTP, interpretadas a la luz de la cortesía verbal, son altamente variables, por lo que son objeto de estudios dialectales (Fernández-Mallat, 2018), diacrónicos (Obediente, 2010) y variacionistas (Roselló, 2018), y su uso está influenciado por factores sociales y pragmáticos (Jang, 2014; Placencia, 2010).

Uno de los trabajos más difundidos sobre este tema, que intenta organizar los sistemas de pronombres usados en las diferentes regiones del mundo hispánico, es el de Fontanella (1999). Su propuesta, que consiste en cuatro sistemas de uso pronominal, es útil porque intenta mostrar la presencia de la variación dialectal en las investigaciones sobre las FTP. La autora presenta, aunque no de manera exhaustiva1, el uso de cada uno de los sistemas en territorios del mundo hispánico y la relación de su uso con aspectos pragmáticos al incorporar en la descripción el eje confianzaformalidad. La autora basa la descripción pragmática de los usos pronominales en el modelo de Brown y Gilman (1960), quienes distinguen el eje de poder, es decir, asimetría en las FTP, y el de solidaridad, en el que hay reciprocidad.

En la síntesis hecha por Carricaburo (2015) de varias investigaciones sobre el tema, Colombia se presenta como un país en el que predomina el tuteo, lo cual se cumple para la región del Caribe, pero en el resto del país hay alternancia, por lo menos, con usted; además, en la costa pacífica, el suroccidente y en parte de la región Andina hay alternancia entre tú, vos y usted (File-Muriel \& Orozco, 2012).

Las investigaciones sobre las FTP en Colombia (Bartens, 2003; Cepeda, 2014, 2017; Jang, 2011, 2014, 2015a, 2015b; Rojas, 2018; Smith, 2014), cuyos resultados serán contrastados con los de esta investigación más adelante, tienen algunos elementos en común que vale la pena resaltar. Todas ellas, después de delimitar una comunidad de habla, parten de cuestionarios para indagar el uso de las FTP según los vínculos con los interlocutores; incluso Cepeda (2014), después de haber utilizado diferentes estrategias de recolección de datos, concluye que los cuestionarios siguen siendo una herramienta idónea, pues

permiten extraer un número amplio de interrelaciones sin tener que generalizar las respuestas del informante (...) y las respuestas, si bien son percepciones de la actuación del hablante, no se alejan de la realidad inmediata de este, razón por la cual permiten ver patrones de conducta de los miembros de un grupo particular. (Cepeda, 2014, p. 195)

$1 \quad$ No incluye sumercé (Cepeda, 2014). 
En estas investigaciones también se indaga por la influencia de factores sociales en la selección de las FTP en los diferentes vínculos con los interlocutores; entre estos factores se encuentran: el sexo, la edad, el nivel educativo, el estrato socioeconómico, la clase social, el lugar de residencia y el origen de los informantes. El análisis de estos elementos permite extraer conclusiones sociolingüísticas sobre la variación o cambios en curso. Uno de los resultados más recurrentes es la diferencia entre hombres y mujeres en el empleo de las FTP. Además, se indaga por las actitudes hacia las FTP para explicar su uso, como es el caso de la conceptualización de tú frente a otras formas como una característica del habla femenina y gay. De igual manera, en estos estudios se interpretan los resultados desde la perspectiva de la cortesía verbal; no solo en lo relativo a la interpretación de las FTP en términos de expresar confianza o formalidad, sino también si el uso indica que la comunidad de habla estudiada, o un grupo dentro de ella, está caracterizado por una preferencia por el trato solidario o de distanciamiento.

Con respecto a la relación de las FTP con la cortesía, Blas (1994) ya había señalado que el modelo más influyente para comprender este fenómeno es el de Brown y Levinson (1987). Esta teoría parte del supuesto de que los hablantes tienen el deseo de proteger su imagen y la de su interlocutor. Para conseguir esto, los participantes despliegan en la comunicación diferentes estrategias que se clasifican en cortesía positiva, si salvaguardan el deseo de ser apreciados por los otros, y cortesía negativa, si cuidan el deseo de autocontrol, dominio propio y el rechazo a imposiciones externas. Entre estas estrategias de cortesía, Brown y Levinson (1987) incluyen la selección pronominal, que hace parte de las manifestaciones gramaticalizadas de dichas estrategias. Diferentes estudios (Serrano, 2000; Jang, 2011) han señalado que, en las comunidades donde hay alternancia entre tú, vos y usted, las formas tú y vos se asocian con la cortesía positiva y usted con la negativa. Haverkate (2000) propuso sustituir los términos de cortesía positiva y cortesía negativa por los de cortesía de solidaridad y cortesía de distanciamiento, respectivamente, pues considera "estos términos más claros y más representativos de los conceptos que pretenden denotar" (Haverkate, 2000, p. 19).

Teniendo en cuenta lo expuesto hasta aquí, esta investigación busca identificar la influencia de las variables sociales en la elección de las FTP, en los diferentes vínculos con los interlocutores, de los hablantes de la ciudad de Cali, Colombia, donde hay alternancia de las formas tú, vos y usted.

La hipótesis principal de la que partimos, de acuerdo con los antecedentes, es que las variables sociales son significativas en la elección de las FTP según el vínculo con los interlocutores.

\section{Metodología}

Esta investigación se inscribe en el marco de la sociolingüística variacionista, que se ocupa de unidades lingüísticas que alternan en el discurso cuyas variantes están relacionadas probabilísticamente con factores lingüísticos y extralingüísticos 
que ayudan a explicar su aparición en el discurso (Blas, 2005). En este sentido, se analiza la relación probabilística de la alternancia entre las formas pronominales vos, tú y usted (variable dependiente) y las variables sociales de sexo, edad, nivel educativo y estrato socioeconómico (variables independientes), variables que han sido significativas en otros estudios de las FTP en el español de Colombia.

Para la definición de las variables, la muestra y el tipo de muestreo en la construcción del corpus, se tomó como referencia el modelo metodológico del Proyecto del Estudio Sociolingüístico del Español de España y América (PRESEEA, s.f.b). La investigación que se presenta en este artículo fue desarrollada dentro del proyecto PRESEEA-Cali.

La población de estudio son los habitantes de la zona urbana de Cali. Esta ciudad, con más de dos millones de habitantes, constituye una comunidad de habla que ya cuenta con algunos estudios que han mostrado algunas características dialectales, además de variación sociolingüística (Ramírez \& Almira, 2016; Ruiz, 2020).

De acuerdo con las directrices de PRESEEA, el muestreo se hizo por cuotas de afijación uniforme, teniendo en cuenta tres variables sociales como criterios de preestratificación: sexo, edad y nivel educativo. La variable sexo comprende las categorías de hombre y mujer; la variable edad está agrupada en tres categorías: generación 1 (20-34 años), generación 2 (35-54 años) y generación 3 (más de 55 años); y la variable nivel educativo se dividió en nivel educativo 1 (primaria), nivel educativo 2 (secundaria) y nivel educativo 3 (superior). Dentro de PRESEEA se estableció que para ciudades de alrededor de dos millones de habitantes la muestra representativa es de 72 informantes. Los criterios de inclusión de los informantes fueron haber nacido en Cali o haber llegado a la ciudad antes de los cinco años y no haber residido, en total, de manera consecutiva o no, más de tres años fuera de la ciudad.

El instrumento de recolección de los datos sobre las FTP fue el "Cuestionario sobre las formas de tratamiento PRESEEA" (PRESEEA, s.f.a). Este cuestionario acompaña la realización de las entrevistas sociolingüísticas que conforman el corpus PRESEEA-Cali. Para la investigación que aquí se presenta se tomó la primera parte del cuestionario que, a través de preguntas directas, indaga por la elección de las formas tú, vos y usted por parte de los informantes dependiendo del vínculo con sus interlocutores. Los vínculos que se exploran son la familia, otro tipo de relaciones (pareja, amigos, conocidos, desconocidos, vecinos) y las ocupaciones de los interlocutores (médico, profesor, cura o pastor, empleada de servicio, mesero, conductor de bus). Cada informante firmó un consentimiento informado que garantiza su anonimato y aceptación de participación y diligenció un formulario con información personal sobre su modo de vida. 
Para el análisis, además de las variables sociales de preestratificación, se agregó la variable de estrato socioeconómico que fue establecida por postestratificación con los datos de la ficha del informante. En Colombia, la estratificación socioeconómica se hace a partir de la clasificación de los inmuebles residenciales y consta de seis categorías (Ley 142 de 1994). Para el análisis, esta variable se agrupó en tres categorías: bajo (estratos 1 y 2), medio (estratos 3 y 4 ) y alto (estratos 5 y 6 ).

De igual manera, para el análisis de las respuestas al cuestionario, se agruparon las categorías de la siguiente manera: relaciones familiares, otro tipo de relaciones I (pareja, amigos), otro tipo de relaciones II (conocidos, desconocidos, vecinos), ocupaciones I (médico, profesor, cura o pastor) y ocupaciones II (empleada del servicio, mesero, conductor de bus). También se analizaron las respuestas de los informantes según si el interlocutor era hombre o mujer.

Por otro lado, en el análisis estadístico se tuvieron en cuenta 71 de los 72 cuestionarios recogidos, pues se descartó uno debido a las inconsistencias de las respuestas del informante y la ausencia de aclaraciones por parte del entrevistador. En primer lugar, se aplicaron medidas de estadística descriptiva para determinar la distribución de la muestra, ya que el estrato socioeconómico no fue una variable de preestratificación, y para establecer la distribución del uso de las FTP en relación con cada una de las categorías de las variables sociales analizadas, para lo cual se utilizaron las frecuencias absolutas y relativas de las respuestas de los participantes. En segundo lugar, se aplicaron métodos de estadística inferencial para comprobar la hipótesis de que las variables sociales son significativas.

Con respecto al análisis de estadística inferencial se utilizó el modelo Probit, un tipo de regresión que usa técnicas similares a la regresión logística, en el programa $\mathrm{R}$ 3.6.3. En cuanto a la significancia se tomaron valores $p$ - menores a 0.05 . En relación con el peso probabilístico se tomó una categoría base para compararla con las otras categorías de la variable. En el caso del sexo la categoría base es hombre; en la edad, la generación 1; en el nivel educativo, el nivel 1; y en el estrato socioeconómico, el estrato bajo. Si los coeficientes son negativos, esto indica que es más probable que la forma de tratamiento que se esté analizando la produzca la categoría base. Así, por ejemplo, comparando las categorías de sexo, un coeficiente negativo indica que es más probable que los hombres, y no las mujeres, produzcan una determinada forma de tratamiento según un vínculo determinado. De esta forma se interpretan las otras categorías de cada variable.

De este modo se obtuvieron los resultados que se presentan a continuación y que se discuten más adelante.

\section{Resultados}

Después de haber sistematizado los cuestionarios e incorporado la variable de postestratificación, la muestra, compuesta por 71 informantes, quedó distribuida como se muestra en la Tabla 1: 
Tabla 1. Distribución de la muestra

\begin{tabular}{lcc}
\hline \multicolumn{1}{c}{ Variable } & Categoría & N \\
\hline Sexo & Hombre & 35 \\
& Mujer & 36 \\
Generación & $20-34$ & 24 \\
& $35-54$ & 24 \\
\multirow{3}{*}{ Nivel educativo } & $>55$ & 23 \\
& Nivel 1 & 24 \\
& Nivel 2 & 24 \\
Estrato & Nivel 3 & 23 \\
socioeconómico & Bajo & 21 \\
& Medio & 44 \\
& Alto & 6 \\
\hline
\end{tabular}

En la primera parte del análisis descriptivo se estableció la distribución general de los datos sobre el uso de las formas de tratamiento pronominales tú, vos y usted. A continuación, en la Tabla 2 se presentan las frecuencias absoluta y relativa de estas tres formas.

Tabla 2. Frecuencia de las FTP

\begin{tabular}{lcc}
\hline & $\mathbf{N}$ & $\mathbf{\%}$ \\
\cline { 2 - 3 } Usted & 1414 & 64,4 \\
Tú & 430 & 19,6 \\
Vos & 351 & 16,0 \\
Total & 2195 & 100,0 \\
\hline
\end{tabular}

De esta manera, los resultados generales muestran un total de 2195 datos. Se puede observar que la FTP más usada es usted, con 1414 casos $(64,4 \%)$, seguida de $t u$, con 430 casos $(19,6 \%)$, y de vos, con 351 (16\%). Hay que resaltar que los hablantes caleños se reconocen como una comunidad voseante y consideran este rasgo como parte de su identidad; sin embargo, en los diferentes vínculos de su vida cotidiana declaran usar preferiblemente las formas tú y usted más que vos. La tendencia en el uso de usted, que representa una importante porción de los casos, indicaría que es una comunidad que se inclina por la cortesía de distanciamiento más que por la de solidaridad. 
En la segunda parte del análisis descriptivo de los datos se observó la distribución del uso de las formas de tratamiento tú, vos y usted dentro de cada una de las variables. La primera en explorarse fue la variable sexo, cuyos resultados se presentan en la Tabla 3:

Tabla 3. Resultados de la variable sexo

\begin{tabular}{lcccccc}
\hline & \multicolumn{2}{c}{ Usted } & \multicolumn{2}{c}{ Tú } & \multicolumn{2}{c}{ Vos } \\
\hline \multirow{2}{*}{ Hombres } & $\mathbf{N}$ & $\mathbf{\%}$ & $\mathbf{N}$ & $\mathbf{\%}$ & $\mathbf{N}$ & \% \\
Mujeres & 646 & 45,7 & 226 & 52,6 & 214 & 61 \\
Total & 768 & 54,3 & 204 & 47,4 & 137 & 39 \\
\hline
\end{tabular}

Se puede ver en esta tabla que las mujeres usan más usted $(54,3 \%)$ que los hombres (45,7\%), mientras que estos usan más tú (52,6\%) y vos (61\%). En esta última forma de tratamiento la diferencia es más acentuada que en las otras. Se puede observar que las mujeres, en el caso de las FTP, favorecen el uso de la cortesía de distanciamiento y desfavorecen la cortesía de solidaridad en las diferentes relaciones. la Tabla 4:

Con respecto a la variable edad, se obtuvieron los resultados presentados en

Tabla 4. Resultados de la variable edad

\begin{tabular}{lcccccc}
\hline & \multicolumn{2}{c}{ Usted } & \multicolumn{2}{c}{ Tú } & \multicolumn{2}{c}{ Vos } \\
\hline $\mathbf{2 0 - 3 4}$ & $\mathbf{N}$ & $\mathbf{\%}$ & $\mathbf{N}$ & $\mathbf{N}$ & $\mathbf{N}$ & \% \\
$\mathbf{3 5 - 5 4}$ & 381 & 26,9 & 161 & 37,4 & 123 & 35 \\
$\mathbf{7 5 5}$ & 482 & 34,1 & 146 & 34 & 133 & 37,9 \\
Total & 551 & 39 & 123 & 28,6 & 95 & 27,1 \\
\hline
\end{tabular}

Estos datos muestran que a medida que aumenta la edad, aumenta el uso de usted, ya que la generación 1 - entre 20 y 34 años $(26,9 \%)$ - usa menos esta forma que la generación 2 - entre 35 y 54 (34,1\%) - y la generación 3 - mayores de 55 (39\%) la usa más que las dos generaciones anteriores. De igual manera, se observa que las generaciones 1 y 2 prefieren el uso de las formas tú (37,4\% y 35\%, respectivamente) y vos (34\% y 37,9\%). Los más jóvenes muestran una tendencia mayor al uso de tú en sus relaciones con respecto a las otras formas de tratamiento. Si a esto se le suma la preferencia de la segunda generación, se infiere que, a pesar de que en general la 
comunidad se inclina por la cortesía de distanciamiento, hay un movimiento hacia la cortesía de solidaridad impulsado por las generaciones más jóvenes.

En relación con la variable de nivel educativo, los resultados fueron los que se muestran en la Tabla 5:

Tabla 5. Resultados de la variable nivel educativo

\begin{tabular}{lcccccc}
\hline & \multicolumn{2}{c}{ Usted } & \multicolumn{2}{c}{ Tú } & \multicolumn{2}{c}{ Vos } \\
\hline \multirow{2}{*}{ Nivel 1 } & $\mathbf{N}$ & $\mathbf{N}$ & $\mathbf{N}$ & $\mathbf{N}$ & $\%$ \\
Nivel 2 & 510 & 36,1 & 150 & 34,9 & 110 & 31,3 \\
Nivel 3 & 455 & 32,2 & 176 & 40,9 & 62 & 17,7 \\
Total & 449 & 31,8 & 104 & 24,2 & 179 & 51 \\
\hline
\end{tabular}

Se puede ver que los hablantes de nivel educativo 1 tienden a usar más usted $(36,1 \%)$, mientras que los de nivel educativo 2 prefieren el uso de tú $(40,9 \%)$ y los de nivel 3 el de vos (51\%) sobre las otras formas de tratamiento. Vemos claramente que a medida que aumenta el nivel educativo disminuye el uso de usted. Estos datos indicarían que el avance de los hablantes de esta comunidad en el nivel educativo tiene una influencia en la elección de las FTP en función de la cortesía de solidaridad, también favorecida por las generaciones jóvenes, como se mostró a propósito de la variable edad.

La última variable que se abordó en el análisis descriptivo fue la de estrato socioeconómico, para la cual se encontró lo que se presenta en la Tabla 6 :

Tabla 6. Resultados de la variable estrato socioeconómico

\begin{tabular}{lcccccc}
\hline & \multicolumn{2}{c}{ Usted } & \multicolumn{2}{c}{ Tú } & \multicolumn{2}{c}{ Vos } \\
\hline \multirow{2}{*}{ Bajo } & $\mathbf{N}$ & $\%$ & $\mathbf{N}$ & $\%$ & $\mathbf{N}$ & $\%$ \\
Medio & 426 & 30,1 & 117 & 27,2 & 79 & 22,5 \\
Alto & 845 & 59,8 & 295 & 68,6 & 237 & 67,5 \\
Total & 143 & 10,1 & 18 & 4,2 & 35 & 10,0 \\
\hline
\end{tabular}


En esta tabla se puede observar que las personas de estrato bajo usan más usted (30,1\%) que las formas tú (27,2\%) y vos (22,5\%), mientras que las personas de estrato medio prefieren el uso de tú $(68,6 \%)$, seguido de vos $(67,5 \%)$ con una frecuencia muy cercana. Por otro lado, los informantes de estrato alto desfavorecen el uso de tú (4,2\%), pero tienen frecuencias similares para usted $(10,1 \%)$ y vos $(10 \%)$. Se puede afirmar que los estratos medios muestran una tendencia a la cortesía de solidaridad, en contraste con los estratos bajos.

Este análisis descriptivo nos ha permitido conocer la distribución del uso de las formas de tratamiento pronominales según las variables sexo, edad, nivel educativo y estrato socioeconómico. En general, aunque es una comunidad con un alto uso de usted, asociado a la cortesía de distanciamiento, se puede observar que la cortesía de solidaridad, agrupada en los usos de tú y vos, es promovida por los hombres, las generaciones 1 y 2 , los niveles educativos 2 y 3 y el estrato socioeconómico medio.

Para el análisis inferencial, partimos de la hipótesis de que existe un aporte significativo de cada una de las variables sociales a la elección de las FTP según el vínculo con los interlocutores dentro del modelo. Las hipótesis de partida se expresan de la siguiente manera:

$$
H 0: B j=0
$$
modelo.

$H a: B j \neq 0$, sea $j$ la representación indicativa de las variables $x$ regresoras del

Regla de decisión: cuando el valor $\mathrm{p}<$ nivel de significancia $(a=0.05)$ se rechaza $H 0$, de lo contrario, no hay suficiente evidencia para hacerlo.

Este modelo se usa para análisis de muestras que no tienen una distribución normal y en las que no hay colinealidad entre las variables dependientes e independientes.

Esto nos permitió saber cuáles de las diferencias constatadas en el análisis descriptivo son pertinentes. Solo se expondrán los resultados de las variables que arrojaron un valor $p<0.05$ en la prueba.

La primera observación fue sobre el uso de las tres FTP en las relaciones familiares. En este caso, las variables sexo y estrato socioeconómico no resultaron significativas, pero sí la edad, para el uso de tú y usted, como se puede observar en la Tabla 7: 
Tabla 7. Resultados del uso de las FTP en las relaciones familiares

\begin{tabular}{|c|c|c|c|}
\hline & $\begin{array}{c}\text { Variable } \\
\text { dependient } \\
e\end{array}$ & $\begin{array}{c}\text { Variable } \\
\text { dependiente }\end{array}$ & $\begin{array}{c}\text { Variable } \\
\text { dependiente }\end{array}$ \\
\hline & Familia Tú & Familia Usted & Familia Vos \\
\hline \multirow[t]{2}{*}{$35-54$} & $-0.960^{* *}$ & 0.748 & 0.782 \\
\hline & $p=0.018$ & $p=0.088$ & $p=0.127$ \\
\hline \multirow[t]{2}{*}{$>55$} & $-0.833^{* *}$ & $1.511^{* * * *}$ & 0.415 \\
\hline & $p=0.043$ & $\mathrm{p}=0.001$ & $p=0.471$ \\
\hline Observations & 71 & 71 & 71 \\
\hline Log Likelihood & -38.901 & -37.236 & -24.979 \\
\hline Akaike Inf. Crit. & 93.801 & 90.471 & 65.958 \\
\hline Note: & $\begin{array}{l}{ }^{* *} \mathrm{p}<0.05 ; \\
\mathrm{p}<<0.01\end{array}$ & & \\
\hline
\end{tabular}

Como se puede observar en la Tabla 7, en el uso de tú en las relaciones de familia (variable dependiente Familia Tú) la variable independiente significativa es la edad, con un valor $p<0.05$. El hecho de que el valor $p$ del coeficiente de la generación 2 (35-54) sea 0.018 y el de la generación $3(>55)$ sea 0.043 , lo cual es un alto grado de significancia y garantiza que los intervalos de los coeficientes de las generaciones 2 y 3 son en su totalidad negativos, implica que es poco probable que usen esta FTP y, en consecuencia, es más probable que la usen las personas de la generación 1 (20-34), que es la variable base para el contraste.

En el caso del uso de usted en este mismo tipo de relaciones (variable dependiente Familia Usted), también se puede ver que la variable significativa es la edad, con un valor $p<0.01$, es decir $p=0.001$, un valor que le otorga una alta certeza estadística al coeficiente positivo, lo que indica que es más probable que este grupo de edad (>55) use usted y es menos probable que esta FTP la usen las personas de la generación 1. En este caso en particular, los datos concuerdan con lo que arrojó el análisis descriptivo, en el que se mostró que las personas de la generación 3 prefieren el uso de usted. 
En síntesis, en el caso de la edad es más probable que la primera generación utilice la FTP tú en contraste con las personas de la segunda y tercera generación, quienes tienden a hacer mayor uso de usted. Para el uso de vos en la familia, en relación con las variables sociales, no se encontró ninguna variable significativa, ya que ninguno de los coeficientes de correlación superó el nivel de significancia estadística del valor $p<0.05$.

Dentro del análisis del uso de las FTP en otro tipo de relaciones, como la pareja y los amigos, no se encontró ninguna variable social significativa. En cambio, en otro tipo de relaciones, como los vínculos con conocidos, vecinos o desconocidos, la variable significativa fue el sexo, como se presenta en la Tabla 8:

Tabla 8. Resultados del uso de las FTP en otros tipos de relaciones

\begin{tabular}{|c|c|c|c|}
\hline & $\begin{array}{c}\text { Variable } \\
\text { dependiente }\end{array}$ & $\begin{array}{c}\text { Variable } \\
\text { dependiente }\end{array}$ & $\begin{array}{c}\text { Variable } \\
\text { dependiente }\end{array}$ \\
\hline & Otras Rel. Tú & $\begin{array}{l}\text { Otras Rel. } \\
\text { Usted }\end{array}$ & Otras Rel. Vos \\
\hline \multirow[t]{2}{*}{ Sexo } & 0.279 & $1.075^{* * *}$ & $-1.104^{* *}$ \\
\hline & $p=0.409$ & $\mathrm{p}=0.010$ & $p=0.062$ \\
\hline Observations & 71 & 71 & 71 \\
\hline Log Likelihood & -36.767 & -27.569 & -16.312 \\
\hline Akaike Inf. Crit. & 89.533 & 71.138 & 48.625 \\
\hline Note: & & & \\
\hline
\end{tabular}

En la Tabla 8 se puede ver que existen diferencias significativas dentro de la variable sexo en el uso de las formas vos y usted con interlocutores con quienes no se tienen vínculos cercanos. Teniendo en cuenta que la categoría base de comparación en esta variable es Hombre, entonces el coeficiente positivo en el uso de usted en otros tipos de relaciones diferentes a las familiares (Otras Rel. Usted), con un valor $p<0.01$, indica que es más probable que las mujeres usen esta forma de tratamiento con este tipo de interlocutores. Por el contrario, el coeficiente negativo en el uso de vos en esos otros tipos de relaciones (Otras Rel. Vos), con un valor $p<0.05$, indica que es más probable que los hombres usen vos con estos interlocutores. 
Con respecto al análisis de la elección de FTP según las ocupaciones de los interlocutores (médico, profesor, cura o pastor, empleada de servicio, mesero, conductor de bus), no se encontró ninguna variable social significativa.

Sí se encontraron diferencias significativas en el análisis de la elección de FTP dependiendo de si el interlocutor es un hombre o una mujer, que aparecen en las tablas como variables dependientes junto con la forma usada. A propósito de esto, se obtuvieron los resultados que se muestran en las tablas 9 y 10 :

Tabla 9. Resultados del uso de las FTP si el interlocutor es una mujer

\begin{tabular}{cccc}
\hline & $\begin{array}{c}\text { Variable } \\
\text { dependiente }\end{array}$ & $\begin{array}{c}\text { Variable } \\
\text { dependiente }\end{array}$ & $\begin{array}{c}\text { Variable } \\
\text { dependiente }\end{array}$ \\
\cline { 2 - 4 } & Mujer Tú & Mujer Vos & Mujer Usted \\
\hline Sexo & -0.289 & -1.008 & $0.722^{* *}$ \\
& $\mathrm{p}=0.491$ & $\mathrm{p}=0.164$ & $\mathrm{p}=0.023$ \\
\hline
\end{tabular}

Tabla 10. Resultados del uso de las FTP si el interlocutor es un hombre

\begin{tabular}{lccc}
\hline & $\begin{array}{c}\text { Variable } \\
\text { dependiente }\end{array}$ & $\begin{array}{c}\text { Variable } \\
\text { dependiente }\end{array}$ & $\begin{array}{c}\text { Variable } \\
\text { dependiente }\end{array}$ \\
\cline { 2 - 4 } & Hombre Tú & Hombre Vos & Hombre Usted \\
\hline Sexo & -0.216 & $-1.754^{* * *}$ & $1.083^{* * * *}$ \\
& $\mathrm{p}=0.716$ & $\mathrm{p}=0.004$ & $\mathrm{p}=0.002$ \\
\hline
\end{tabular}

En la Tabla 9 se evidencia que cuando el interlocutor es una mujer (en este caso como variable independiente), las mujeres (que están incluidas en la variable independiente "sexo" como locutoras) prefieren usar la forma usted (coeficiente $0.722, \mathrm{p}=0.023$ ). A pesar de que los resultados para las otras dos formas no tienen valores $p$ significativos, se puede apreciar que los hombres, cuando el interlocutor es una mujer, tienden a usar las formas vos y tú.

Por otro lado, en la Tabla 10 se puede observar que, de manera significativa, cuando el interlocutor es un hombre, las mujeres usan preferiblemente usted 
(coeficiente 1.083, $\mathrm{p}=0.002$ ), mientras que los hombres tienden a usar más vos (coeficiente $-1.754, \mathrm{p}=0.004$ ) con otros hombres. Aunque los datos de la elección de tú no son significativos, se puede ver que los hombres también tienden a optar por esta forma en lugar de usted.

De esta manera, se concluye que las mujeres prefieren el trato de usted con hombres y mujeres, mientras que los hombres con otros hombres prefieren el trato de vos y con mujeres podrían preferir el trato de tú o vos en lugar de usted.

Los resultados expuestos en este apartado permitieron conocer la distribución del uso de las FTP en general y en relación con las variables sociales. De igual manera, se mostró que la comunidad en general tiene una tendencia a la cortesía de distanciamiento, pues en un alto porcentaje, que supera los porcentajes de las dos otras formas de tratamiento juntas, los informantes declaran usar usted. Asimismo, se vio que, en los datos generales, los hombres usan más tú que las mujeres, lo que llevaría a plantear que no hay una estigmatización de esta forma por parte de los hombres, hipótesis que deberá ser corroborada con la incorporación al análisis de las preguntas sobre aspectos actitudinales de la elección de las FTP.

Se corroboró la hipótesis nula para las variables nivel educativo y estrato socioeconómico, mientras que la hipótesis alternativa se corroboró para las variables edad y sexo con algunas variables dependientes. En este sentido, se encontró que la variable sexo sí es significativa para la elección de formas pronominales en las relaciones con sus interlocutores diferentes a las familiares, específicamente aquellas en las que hay más distancia social, y tiene un peso importante en el uso de las FTP dependiendo del sexo del interlocutor. De igual manera, la variable edad resultó significativa en el tratamiento pronominal en las relaciones familiares.

En el siguiente apartado de discusión se desarrollarán algunos elementos aportados por otras investigaciones sobre las FTP en relación con los hallazgos de nuestro análisis.

\section{Discusión}

Con respecto a otros estudios sobre las formas de tratamiento pronominales en Colombia se pueden establecer algunas convergencias y divergencias con los hallazgos de esta investigación. Para empezar, se puede contrastar la forma de tratamiento más usada en cada una de las comunidades estudiadas. En este sentido, hay una coincidencia con los resultados obtenidos en Bogotá, Medellín y Cali. En estas ciudades la forma más usada es usted, tal como lo muestran el estudio de Cepeda (2014) en Bogotá, en el que la frecuencia de uso de esta forma es del 59,6\%; el de Jang (2014) en Medellín y en zonas semirrurales y rurales de Antioquia, donde el uso de usted prevalece tanto en las relaciones familiares como en otro tipo de relaciones; y los datos de Cali, donde encontramos una frecuencia de 64,4\%. De igual manera, en el estudio hecho en Sogamoso (Rojas, 2018) se encontró que la forma más usada en el análisis de cada una de las variables sociales fue $u$ sted. 
Estos datos indican que en los subdialectos caucano-valluno, antioqueñocaldense y cundiboyacense (Ruiz, 2020), parece primar la cortesía de distanciamiento, hipótesis que debe corroborarse con datos de otras poblaciones pertenecientes a estos subdialectos. No obstante, los datos disponibles de ciudades representativas de estos subdialectos permiten afirmar que, por lo menos en estas regiones de Colombia, no se constata la tendencia que han señalado investigadores como Blas (2005) hacia el predominio del tratamiento de solidaridad sobre el de distanciamiento. Atendiendo a la evolución del uso de las FTP en el español peninsular y el de América, en la que el progresivo paso de usted a tú como forma más frecuente en España y México, o a vos en el caso de Argentina, se ha interpretado como una práctica lingüística innovadora (Jang, 2014), se puede decir que estas regiones colombianas son conservadoras.

Por otro lado, en los estudios sobre FTP hechos en Colombia se ha mostrado que la variable sexo es importante en la elección de la forma empleada. Así, en Bogotá (Cepeda, 2014) los hombres usan más usted que las mujeres (65,4\% frente a 53,6\%) y las mujeres tutean más que los hombres (36,3\% frente a $26 \%$ ). Estos patrones de uso ya habían sido observados también por Bartens (2003). En Tunja (Smith, 2014), - dejando a un lado los datos de sumercé, que no serían comparables -, los hombres también usan más usted que las mujeres (42\%-40\%) y estas emplean más tú que los hombres (35\%-30\%), tendencia que también se encontró en Sogamoso (Rojas, 2018). En Medellín (Jang, 2011), en general, son las mujeres quienes usan más usted y tú que los hombres, mientras que estos usan más vos. En Cali, como se expuso en los resultados, también son las mujeres quienes usan más usted (54,3\%), pero los hombres usan más tú $(52,6 \%)$ y vos (61\%). Estos datos de la comunidad de habla de Cali difieren de los encontrados en Bogotá, Tunja y Sogamoso, y coinciden en parte con los de Medellín, lo que acercaría más el habla caleña al habla medellinense que a la de las otras ciudades. Una futura vía de investigación podría indagar por estas diferencias dialectales para establecer si son perceptibles.

A propósito de estas diferencias de uso entre hombres y mujeres, Smith (2014) sostiene que la elección de usted podría asociarse al mantenimiento de normas conservadoras y más prestigiosas tradicionalmente, relacionadas con la cortesía de distanciamiento. En este sentido, acogiendo lo que se dijo antes sobre la evolución de las FTP en español, se podría decir que en Bogotá, Tunja y Sogamoso las mujeres son innovadoras en el trato y los hombres conservadores, mientras que en Medellín y Cali las mujeres serían más conservadoras y los hombres innovadores. Sin embargo, esto debe ser investigado con más detalle porque en diferentes comunidades de habla las FTP que tradicionalmente expresan cortesía de distanciamiento pueden ser usadas como cortesía de solidaridad (Cepeda, 2014) y viceversa.

Sobre la variable edad, tanto en Bogotá como en Sogamoso se encontró que, aunque la generación 1 usa más tú que las otras generaciones, la forma más usada frente a tú en todas las generaciones es usted. En Cali, la generación 1 usa más tú y vos que usted, mientras que la generación 3 usa más esta última forma. El contraste de estos datos permite inferir que, en el habla caleña, contrario a lo que sucede en 
las otras dos comunidades de habla, las generaciones jóvenes son innovadoras en el tratamiento pronominal y la tercera generación es conservadora, lo cual se atribuye a que los hablantes de este grupo son promotores de las normas más tradicionales (Cepeda, 2014).

Esta tendencia se corroboró en el análisis inferencial, en el que se encontró que en las relaciones familiares la generación 1 prefiere el uso de tú y la generación 3 se inclina por el uso de usted. Sería interesante avanzar en otros estudios que exploren si estos resultados en Cali corresponden a un cambio en marcha sobre el paso de una tendencia de tratamiento de distanciamiento a uno solidario, como ha ocurrido en otras variedades del español (Jang, 2014).

Los datos de Sogamoso (Rojas, 2018) en relación con la variable de nivel educativo muestran que todos los niveles usan más la forma usted. Sin embargo, los niveles educativos 2 y 3 tienden a usar más tú que el nivel 1 . En Cali se ve claramente la preferencia de los niveles educativos 2 y 3 por las formas de tratamiento solidario vos y tú en lugar de la forma de distanciamiento, que es la más usada por el nivel educativo 1. En este caso, se puede ver que los grupos más prestigiosos y aquellos que jalonan los cambios lingüísticos, como es el caso de los grupos intermedios de escolaridad (Blas, 2005), son los que se inclinan por el tratamiento solidario en Cali, lo cual es otro aliciente para investigar si hay indicios de un cambio en marcha en el estilo de tratamiento de la comunidad.

La variable estrato socioeconómico también es abordada en los estudios de Bogotá y Medellín. En ambos casos se pudo observar que, en general, todos los estratos socioeconómicos usan más usted que tú y vos (este último en el caso de Medellín); estas dos últimas formas van aumentando a medida que se incrementa el estrato socioeconómico. En Cali pasa algo similar. Aunque la tendencia en los estratos socioeconómicos altos no es clara, sí hay una diferencia marcada entre los estratos bajos y los medios en el sentido de que estos últimos usan en mayor medida las formas tú y vos. Una vez más se puede apreciar que, a pesar del predominio de la forma de distanciamiento en la comunidad, los grupos de prestigio son los más innovadores hacia el tratamiento solidario.

Uno de los resultados más interesantes en algunos estudios de las FTP en Colombia es la influencia del sexo del interlocutor en la elección del pronombre de tratamiento. En el caso de Antioquia (Jang, 2015a), en el que se incluye el análisis de Medellín, se encontró que los hombres prefieren usar vos o usted con otros hombres en lugar de tú. En el caso de Bogotá (Cepeda, 2014), se observó que las mujeres tratan mayoritariamente de tú a hombres y mujeres; en cambio, los hombres tratan de tú a las mujeres y de usted a los hombres. Estos resultados llevaron a ambos investigadores, Jang (2015b) y Cepeda (2017) a indagar sus causas. En los dos estudios se llegó a la conclusión de que estas diferencias se deben a la estigmatización del uso de tú por parte de los hombres, pues conlleva un matiz feminizante (Jang, 2015b) y es propio del habla femenina y del habla gay (Cepeda, 2017). En Cali (ver tablas 9 y 
10) los resultados difieren con respecto a las mujeres, pues en esta comunidad ellas prefieren el uso de usted tanto con hombres como con mujeres; pero los datos de los hombres muestran un derrotero parecido al de Bogotá y Medellín, ya que ellos, cuando el interlocutor es un hombre, significativamente prefieren usar vos en lugar de tú. En el caso de Cali, los hombres tratan de manera solidaria a sus interlocutores sin importar el sexo y las mujeres lo hacen con cortesía de distanciamiento. De igual manera, parece ser que los hombres siguen evitando el tuteo con otros hombres. Habría que indagar si también en este caso se trata de una estigmatización de esta forma de tratamiento.

Aquí no podemos avanzar en esta última vía, porque en este estudio no se analizaron aspectos actitudinales en relación con las formas de tratamiento. Para indagar en el estatus del tuteo en esta comunidad se deben analizar preguntas sobre si el uso de tú se considera formal o informal, refinado o no, y si se asocia con el habla femenina o masculina.

El recorrido que hemos hecho en este artículo permitió conocer datos que no se tenían sobre las FTP en el español hablado en Cali, contrastarlos con otras variedades de habla para ver tendencias más generales en el español de Colombia 0 particulares de la comunidad de habla caleña y su análisis en términos de la cortesía de solidaridad o de distanciamiento. De igual manera, se identificaron otras preguntas de investigación que pueden ser desarrolladas en posteriores estudios sobre similitudes y diferencias dialectales, la indagación por un posible cambio en marcha desde la cortesía de distanciamiento a la cortesía de solidaridad y el estudio de las actitudes de los hablantes sobre el uso de las FTP de acuerdo con el sexo del interlocutor.

\section{Conclusiones}

Cali es una comunidad de habla en la que prima el uso de usted sobre las formas tú y vos. Esto indica que hay una predominancia de la cortesía de distanciamiento, lo que la alinea con otras comunidades en las que se ha comprobado esta misma tendencia, como Bogotá, Medellín y Sogamoso. En este sentido, se puede catalogar como una comunidad conservadora en el uso de las formas de tratamiento pronominales.

Las mujeres son quienes usan más la forma de tratamiento de distancia usted y los hombres prefieren las formas de solidaridad tú y vos. En consecuencia, en el habla de Cali las mujeres son conservadoras y los hombres innovadores en el trato pronominal. Este aspecto aproxima la variedad caleña a la medellinense y la distancia de las variedades de Bogotá, Tunja y Sogamoso.

Aunque la comunidad en general tiende a la cortesía de distanciamiento, algunos grupos impulsan el uso de las formas de tratamiento de solidaridad. Así, el uso de los pronombres tú y vos es promovido por los hombres, las dos generaciones más jóvenes, los niveles educativos más altos y los estratos socioeconómicos medios. Al tratarse de grupos prestigiosos o que jalonan los cambios en las comunidades, 
podría haber un cambio en marcha hacia la cortesía de solidaridad, como ha ocurrido en otras variedades del español.

La variable edad es significativa en el tratamiento pronominal en las relaciones familiares. También resultó significativa la variable sexo en la elección de formas pronominales en las relaciones diferentes a las familiares, especialmente aquellas en las que hay mayor distancia social, y tiene un peso importante en el uso de las FTP dependiendo del sexo del interlocutor.

Con respecto a la elección de la forma pronominal según el sexo del interlocutor, en la que los hombres evitan usar tú con interlocutores del mismo sexo, Cali va en la misma vía de lo que se ha encontrado en Medellín y Bogotá, comunidades en las que se ha percibido una estigmatización del tuteo por parte de los hombres

Quedan por explorar, en futuros estudios, los contrastes dialectales más amplios sobre las formas de tratamiento pronominales, la indagación sobre aspectos actitudinales, como el registro y el estilo de habla al que asocian los caleños las FTP, y la verificación de un posible cambio en marcha desde la cortesía de distanciamiento hacia la cortesía de solidaridad en la comunidad de habla de Cali.

\section{Referencias}

Bartens, A. (2003). Notas sobre el uso de las formas de tratamiento en el español colombiano actual. En Comunicación presentada en el Coloquio Pronombres de segunda persona y formas de tratamiento en las lenguas de Europa. Instituto Cervantes, París, Francia. https://cvc.cervantes.es/lengua/coloquio paris/ponencias/bartens.htm

Bertolotti, V. (2015). A mí de vos no me trata ni usted ni nadie: sistemas e historia de las formas de tratamiento en la lengua española en América. Universidad Nacional Autónoma de México, Universidad de la República.

Blas, J. (1994). Los pronombres de tratamiento y la cortesía. Revista de Filología de la Universidad de La Laguna, (13), 7-35.

Blas, J. (2005). Sociolingüística del español: desarrollos y perspectivas en el estudio de lengua española en contexto social. (2. ${ }^{\mathrm{a}}$ ed.). Cátedra.

Brown, P., \& Levinson S. C. (1987). Politeness. Some Universals in Language Usage. Cambridge University Press.

Brown, R., \& Gilman, A. (1960). The Pronouns of Power and Solidarity. In T. A. Sebeok (ed.), Style in Language (pp. 253-276). The M.I.T. Press.

Carricaburo, N. (2015). Las fórmulas de tratamiento en el español actual (2a ed.). Arco Libros. 
Cepeda, C. (2014). Usted, tú, sumercéy vos: formas pronominales de tratamiento en el español de Bogotá (Colombia). (Tesis de Maestría). Universidad Nacional Autónoma de México, Ciudad de México, México. http://132.248.9.195/ ptd2014/octubre/0721377/Index.html

Cepeda, C. (2017). ¿Tú o usted? Estigmatización del tuteo en Bogotá. Anuario de Letras. Lingüística y Filología, 5(2), 35-65. https://revistas-filologicas.unam. $\mathrm{mx} /$ anuario-letras/index.php/al/article/view/1463/1660

Fernández-Mallat, V. (2018). Alternancia y variación de formas verbales tuteantes y voseantes en el español de santiaguinos: estudio de caso basado en un corpus conversacional. Boletín de Filología, 53(1), 63-82. https://boletinfilologia. uchile.cl/index.php/BDF/article/view/50637

File-Muriel, R. J., \& Orozco, R. (Eds.) (2012). Colombian Varieties of Spanish. Iberoamericana-Vervuert.

Fontanella, M. (1999). Sistemas pronominales de tratamiento usados en el mundo hispánico. En I. Bosque \& V. Demonte (dirs.), Gramática descriptiva de la lengua española (vol. 1) (pp. 1399-1425). Espasa.

Haverkate, H. (2000). Estrategias de cortesía. Análisis intercultural. Forma y Función, (13), 17-30. https://revistas.unal.edu.co/index.php/formayfuncion/article/ view/17180

Jang, J. S. (2011). Fórmulas de tratamiento pronominales en los jóvenes universitarios de Medellín (Colombia) desde la óptica sociopragmática: estrato socioeconómico y sexo. Íkala, Revista de Lenguaje y Cultura, 15(3), 43-116. https://revistas.udea.edu.co/index.php/ikala/article/view/7791

Jang, J. S. (2014). La frecuencia del uso del usted en tres zonas del departamento de Antioquia (Colombia). Forma y Función, 27(1), 45-65. https://doi. org/10.15446/fyf.v27n1.46943

Jang, J. S. (2015a). La frecuencia del tuteo en tres zonas del departamento de Antioquia (Colombia): influencia de la zona urbana/rural. Forma y Función, 28(1), 1129. https://doi.org/10.15446/fyf.v28n1.51969

Jang, J. S. (2015b). Matiz feminizante del tuteo y el futuro del voseo en el departamento de Antioquia (Colombia). Estudios Filológicos, (56), 85-99. https://doi. org/10.4067/S0071-17132015000200005

Obediente, E. (2010). Visión diacrónica y dialectal de las formas de tratamiento en los Andes venezolanos. En C. Borgonovo, M. Español-Echevarría \& P. Prévost (eds.), Selected Proceedings of the 12th Hispanic Linguistics Symposium (pp. 87-96). https://www.lingref.com/cpp/hls/12/paper2408.pdf 
Placencia, M. (2010). El estudio de formas de tratamiento en Colombia y Ecuador. En M. Hummel, B. Kluge \& M. Vásquez (eds.), Formas y fórmulas de tratamiento en el mundo bispánico (pp. 341-373). Colegio de México.

Proyecto del Estudio Sociolingüístico del Español de España y América PRESEEA (s.f.a). Cuestionario sobre las formas de tratamiento PRESEEA. https:// preseea.linguas.net/Portals/0/Metodologia/Cuestionario\%20FT.pdf

Proyecto del Estudio Sociolingüístico del Español de España y América PRESEEA (s.f.b) Guías PRESEEA de Estudios. https://preseea.linguas.net/Metodolog\%C3\%ADa. aspx

Ramírez, A. \& Almira, A. (2016). Variación del fonema /s/ en contextos de juntura de palabra en el español caleño: una mirada sociolingüística. Lenguaje, 44(1), 11-33. https://doi.org/10.25100/lenguaje.v44i1.4628

Rojas, J. (2018). Formas y fórmulas de tratamiento en el español hablado en Sogamoso (Colombia). (Tesis de Maestría). Universidad Pedagógica y Tecnológica de Colombia, Tunja, Colombia. http://repositorio.uptc.edu.co/ handle/001/2343

Roselló, J. (2018). Las formas de tratamiento en el corpus PRESEEA-Valencia. Un estudio sociolingüístico. Círculo de Lingüística Aplicada a la Comunicación, 76, 241-260. https://doi.org/10.5209/CLAC.62507.

Ruiz, N. (2020). El español de Colombia. Nueva propuesta de división dialectal. Lenguaje, 48(2), 160-195. https://doi.org/10.25100/lenguaje.v48i2.8719

Serrano, M. (2000). La producción de la cortesía verbal y la deíxis sociocomunicativa. Oralia, (3), 199-219.

Smith, G. (2014). Formas pronominales de tratamiento y cortesía en el habla de Tunja, Colombia. Folios, (39), 31-49. https://doi. org/10.17227/01234870.39folios31.49 